\title{
ON HARMONIC AND ANALYTIC FUNCTIONS
}

\section{FRANTIŠEK WOLF}

If we study the behavior of a harmonic function on the boundary of the unit circle along an arc $\alpha<\theta<\beta$, it is sometimes of advantage, if the function behaves in the simplest possible way outside this arc. This problem of isolating the singular arc can easily be solved for a harmonic function which is bounded in the unit circle. For such a function can be expressed by means of a Poisson integral

$$
u(r, \theta)=\frac{1}{2 \pi} \int_{-\pi}^{\pi} \frac{1-r^{2}}{1-2 r \cos (\theta-\vartheta)+r^{2}} u(1, \vartheta) d \vartheta
$$

and then

$$
v(r, \theta)=\frac{1}{2 \pi} \int_{\alpha}^{\beta} \frac{1-r^{2}}{1-2 r \cos (\theta-\vartheta)+r^{2}} u(1, \vartheta) d \vartheta
$$

is known to behave in the same way as $u(r, \theta)$ in the neighborhood of the arc $(\alpha, \beta)$-in fact the difference of the two functions tends uniformly to zero inside the arc-and $v(r, \theta)$ can be extended so as to make it harmonic and equal to zero on the rest of the circumference.

It is equally easy to solve the problem for a harmonic function $u(r, \theta)$ which is $O\left(1 /(1-r)^{n}\right)$ near the circumference and is, therefore, the $(n+2)$ nd derivative of a harmonic function, bounded in $r \leqq 1$.

The purpose of this paper is to show that the problem can be solved for any function harmonic in $r<1$. The result can be generalized to any domain which can be represented conformally on the unit circle.

THEOREM. If $u(r, \theta)$ is a function, harmonic in the unit circle, then, given the arc $(r=1, \alpha<\theta<\beta)$, there is a function $v(r, \theta)$ harmonic in $r<1$, such that $u(r, \theta)-v(r, \theta)$ can be extended across the arc $(\alpha, \beta)$ so as to make it harmonic and zero on the arc; and $v(r, \theta)$ is harmonic and zero along the rest of the circumference.

Proof. (i) If

$$
u(r, \theta)=\sum_{1}^{\infty} r^{n}\left(a_{n} \cos n \theta+b_{n} \sin n \theta\right)
$$

we can find a nonincreasing sequence $\epsilon_{n} \rightarrow 0$, such that

$$
\left|a_{n}\right|,\left|b_{n}\right| \leqq\left((1 / 2) e^{\epsilon_{n} n}-2\right) / n^{2}
$$

Received by the editors October 5, 1942. 
We have indeed

$$
r^{n}\left\{\begin{array}{l}
a_{n} \\
b_{n}
\end{array}\right\}=\frac{1}{2 \pi} \int_{0}^{2 \pi} u(r, \theta)\left\{\begin{array}{l}
\cos n \theta \\
\sin n \theta
\end{array}\right\} d \theta .
$$

If we put $r=e^{-\epsilon}$, we find that whatever $\epsilon>0$ we select, $\left|a^{n}\right|,\left|b^{n}\right|$ $\leqq U e^{\epsilon n}$ for all $n$. Hence

$$
\lim \sup \frac{\log \left|a_{n}\right|}{n} \leqq 0, \quad \lim \sup \frac{\log \left|b_{n}\right|}{n} \leqq 0
$$

and, therefore,

$$
\epsilon_{n}=\max _{k=n, n-1, \cdots}\left[0, k^{-1} \log \left(2\left|a_{k}\right| \cdot k^{2}+4\right), k^{-1} \log \left(2\left|b_{k}\right| \cdot k^{2}+4\right)\right]
$$

will satisfy our requirements.

(ii) There exists an entire function $\phi(z)$ such that $\phi(z)=O\left(e^{\epsilon|z|}\right)$ for any $\epsilon>0$ and sufficiently large $|z|, \overline{\phi(z)}=\phi(\bar{z}), \phi(z)=\phi(-z), \phi(0)=0$ and

$$
\left(\left|a_{n}\right|+\left|b_{n}\right|\right) / \phi(n) \leqq 2 / n^{2},
$$

for all $n$ so large that $2 \epsilon_{n} \leqq 1$. We define $m_{n}$ as the least integer such that $\sum_{k>m_{n}} n^{2 k} /(2 k) ! \leqq 1$. Then

$$
\phi(z)=\sum_{1}^{m_{1}} \frac{\left(\epsilon_{1} z\right)^{2 k}}{(2 k) !}+\sum_{m_{1}+1}^{m_{2}} \frac{\left(\epsilon_{2} z\right)^{2 k}}{(2 k) !}+\cdots=\sum_{k=1}^{\infty} \frac{\left(\delta_{k} z\right)^{2 k}}{(2 k) !} .
$$

This function satisfies our requirements. Evidently, it is sufficient to show that (2) is satisfied.

Since

we have

$$
\sum_{k>m_{n}}^{\infty} \frac{\left(\delta_{k} n\right)^{2 k}}{(2 k) !} \leqq \epsilon_{n+1}^{2 m_{n}} \sum_{k>m_{n}}^{\infty} \frac{n^{2 k}}{(2 k !)} \leqq \epsilon_{n+1}^{2 m_{n}}
$$

$$
\begin{aligned}
\phi(n) & \geqq \sum_{1}^{m_{n}} \frac{\left(\delta_{k} n\right)^{2 k}}{(2 k) !}-\epsilon_{n}^{2 m_{n}} \geqq \sum_{1}^{\infty} \frac{\left(\epsilon_{n} n\right)^{2 k}}{(2 k) !}-2 \epsilon_{n}^{2 m_{n}} \\
& =(1 / 2)\left(e^{\epsilon_{n} n}+e^{-\epsilon_{n} n}\right)-1-2 \epsilon_{n}^{2 m_{n}}
\end{aligned}
$$

and, for $2 \epsilon_{n} \leqq 1$, this yields, by (1),

$$
\left|a_{n}\right| n^{2},|b| n^{2} \leqq \phi(n)
$$

(iii) From (2) it follows that

$$
U(r, \theta)=\sum_{1}^{\infty} r^{n} \frac{a_{n} \cos n \theta+b_{n} \sin n \theta}{\phi(n)}
$$


is a harmonic function, continuous in the closed unit circle. Hence we can express it by means of a Poisson integral

$$
\begin{aligned}
U(r, \theta) & =\frac{1}{2 \pi} \int_{0}^{2 \pi} U(1, \vartheta) \frac{\left(1-r^{2}\right) d \vartheta}{1-2 r \cos (\vartheta-\theta)+r^{2}} \\
& =\frac{1}{2 \pi}\left(\int_{\alpha}^{\beta}+\int_{(0,2 \pi)-(\alpha, \beta)}\right) U(1, \vartheta) \frac{\left(1-r^{2}\right) d \vartheta}{1-2 r \cos (\vartheta-\theta)+r^{2}} \\
& =V(r, \theta)+W(r, \theta) .
\end{aligned}
$$

The harmonic function $V(r, \theta)$ expressed by the first integral can be extended across the arc complementary to $(\alpha, \beta)$, so that it becomes harmonic and zero on the arc. For $W(r, \theta)$ the same is true with respect to the arc $(\alpha, \beta)$ itself. We see that the function $V(r, \theta)$ bears to $U(r, \theta)$ the same relation as the function $v(r, \theta)$, required by the theorem, should bear to $u(r, \theta)$.

(iv) Wigert's theorem asserts that if $\phi(z)$ is an entire function satisfying the first condition in (ii), then

$$
f(z)=\sum_{0}^{\infty} \phi(n) z^{n}
$$

is a function having its only singular point at $z=1$. We get easily

$$
f\left(e^{w}\right)=\sum_{k=1}^{\infty} \frac{\left(\delta_{k}\right)^{2 k}}{(2 k) !} \frac{d^{2 k}}{d w^{2 k}}\left(\frac{1}{1-e^{w}}\right) .
$$

In a proof of the above theorem, given by Bieberbach, ${ }^{1}$ it is shown that this last series converges uniformly in a neighborhood of any point different from $w=0$. Therefore, we have

$$
\begin{aligned}
f\left(e^{-w}\right) & =\sum_{1}^{\infty} \frac{\delta_{k}^{2 k}}{(2 k) !} \frac{d^{2 k}}{d w^{2 k}}\left(\frac{1}{1-e^{-w}}\right)=-\sum_{1}^{\infty} \frac{\delta_{k}^{2 k}}{(2 k) !} \frac{d^{2 k}}{d w^{2 k}}\left(\frac{1}{1-e^{w}}\right) \\
& =-f\left(e^{w}\right) .
\end{aligned}
$$

For $w=i \theta$, we get $(1 / 2)\left(f\left(e^{i \theta}\right)+f\left(e^{-i \theta}\right)\right)=R\left[f\left(e^{i \theta}\right)\right]=0$. Hence $K(r, \theta)$ $=\sum_{1}^{\infty} r^{n} \phi(n) \cos n \theta$ is a function, harmonic in the whole plane except at $r=1, \theta=0$, and is zero for $r=1, \theta \neq 0$.

(v) Using Parseval's identity, we find without difficulty that

$$
\frac{1}{\pi} \int_{-\pi}^{\pi} K(r, \theta-x) U(1, x) d x=\sum r^{n}\left(a_{n} \cos n \theta+b_{n} \sin n \theta\right)=u(r, \theta) .
$$

\footnotetext{
${ }^{1}$ Lehrbuch der Funktionentheorie, vol. 2, pp. 288-292.
} 
Further

$v(r, \theta)=\frac{1}{\pi} \int_{-\pi}^{\pi} K(r, \theta-x) V(1, x) d x=\frac{1}{\pi} \int_{\alpha}^{\beta} K(r, \theta-x) V(1, x) d x$

is clearly a function, harmonic in $r<1$ and it is easy to deduce from the properties of $K(r, \theta)$ and $V(1, \theta)$ that $v(r, \theta)$ satisfies all the conditions of the theorem.

COROLlary. If $f(z)$ is analytic in $|z|<1$, then, given the arc $(|z|=1$, $\alpha<\arg z<\beta)$, there is a function $g(z)$ analytic in $|z|<1$ and on the arc of $|z|=1$ complementary to $(\alpha, \beta)$, such that $f(z)-g(z)$ can be extended analytically across $(\alpha, \beta)$.

University OF CALIFornia

\section{ON THE COMPLEX ZEROS OF THE BESSEL FUNCTIONS}

E. HILLE AND G. SZEGÖ

1. Introduction. Various proofs have been given for the following classical theorem of A. Hurwitz:

THEOREM 1. The entire function

$$
z^{\beta / 2} J_{-\beta}\left(2 z^{1 / 2}\right)=\sum_{m=0}^{\infty} \frac{(-z)^{m}}{m !} \frac{1}{\Gamma(m+1-\beta)}
$$

has precisely $[\beta]$ nonpositive zeros. Here $J_{-\beta}$ is the Bessel function of order $-\beta$ and $\beta \geqq 0$.

In case $\beta$ is an integer these nonpositive zeros are all at the origin; in case $\beta$ is not an integer and $[\beta]$ is odd there is precisely one negative zero and we have $\frac{1}{2}([\beta]-1)$ pairs of conjugate complex zeros; in case $\beta$ is not an integer and $[\beta]$ is even there are $\frac{1}{2}[\beta]$ pairs of conjugate complex zeros.

Most of the proofs for this theorem (see the papers $[2,4,6,7,9]$ of the Bibliography at the end of the text) make use of polynomial approximations of the Bessel function. The present proof follows the same line by obtaining the Bessel function as the limit of Laguerre polynomials. The study of the complex zeros of these polynomials is 1942.

Presented to the Society, September 9, 1942; received by the editors September 14, 